\title{
The Analysis of Computer Models of Wetland Ecological Changes Based on GIS Technology
}

\author{
Liwei Liu* \\ College of Biology and the Environment, Nanjing Forestry University, Nanjing, JiangSu, China
}

\begin{abstract}
Wetlands are important to earth environment and ecosystems. With the rapid development of computer technology, establishing computer models of wetland analysis, especially models based on wetland monitoring, has become an important way to protect wetlands. Wetland monitoring needs data collection, entry, retrieval and analysis of a large number of representative wetlands. GIS technology application and wetland computer model can significantly improve the work efficiency. This paper provides a brief introduction of GIS, and an overview on wetland computer models based on GIS. This paper also studies the GIS application in data management model of wetland monitoring. Through the examples of GIS application in wetland monitoring analysis, the final GIS applications in wetland monitoring and computer models are put forward.
\end{abstract}

Keywords: Computer model, GIS technology, Wetland change, Wetland ecology.

\section{INTRODUCTION}

Wetlands are unique marine resources located in the transition zone of terrestrial ecosystem and marine ecosystem; wetlands are also the most active interactions of land, ocean and atmosphere. They are seen as the buffer of global environmental change. Wetland areas do not only serve ecosystems and supply resources, but also provide rich lands, salt and other resources to people for industry, tourism, biotechnology. They can regulate climate, take invasive accommodate nutrients under control, and purify pollutants. These important regulatory functions bring wetlands the title of "ocean kidney". But with the economic growth, as well as the increasing pressure of population and human activities, wetlands are now under growing influences, which lead to the increasing pressure suffered by the environment. Coastal construction and irrational resource exploitation cause the degradation of wetland ecosystems, the rise of sea level, marine pollution, coastal land salinization, red tide, habitats deterioration, biodiversity loss, a series of land subsidence in coastal wetland, and other environmental problems. The loss of wetland ecological functions and the increasing scarcity of resources cause ecological damage to the environment, which will severely restrict the economic development in wetland areas. Therefore, analyzing the dynamic change pattern of wetlands, and establishing wetland ecological database, have become important issues in current wetland research [1].

Currently, computer technology has been widely used in wetland resources information management systems with different sizes and different functions. These applications have achieved good economic, social and ecological benefits.

*Address for correspondence to this author: College of Biology and the Environment, Nanjing Forestry University, Nanjing, JiangSu, China

E-mail: 76560136@qq.com
With the urgent need for the study of regional and ecological protection, the techniques of comprehensive applications technology, GIS technology, environmental engineering, database technology, information management system and decision support system in wetland protection are becoming more mature. Geographical study, however, needs to deal with a wide range of complex and diverse natural conditions, as well as the traffic inconvenience. The traditional survey research methods require a huge amount of human, material and financial resources; the survey cycle is relatively long. But by using GIS technology, the basic situation of an area can be understood in a relatively short period of time with lower costs on human and financial resources. In this way, researchers can evaluate the status and trends of wetland usage, and provide scientific foundation for wetland resources optimization and protection in the future. [2]

\section{RESEARCH MATERIAL AND METHODS}

\subsection{Foreign Research Status of Wetland Models}

In 1970s, researcher E.F. Codd proposed the relational wetland model. Since then, researchers have studied the issue of database. A number of foreign countries have developed practical engineering systems which can basically satisfy their requirements. Relatively well-known examples are as follows. American company launched Primavera P3 (Primavera Project Planner, referred to as P3), which can be used in system planning, project management, encoding settings, detailed planning, schedule calculation and statistical analysis. Carnegie Mellon University developed a CALLISTO systems on simulation, monitoring and scheduling to manage large projects. Microsoft company developed Microsoft schedule management software, which can help users to establish project schedule, analyze and optimize the schedule, implement and change the plan, as well as control and manage targets. CAD /CAM center of Norwegian Insti- 
tute developed industrial applications and database management systems TORNADO. The system provides users with a very flexible mesh model definition language. TORNADO Version5.0 also supports the definition of complex objects and treatment, and increases dynamic arrays in data types. [3]

\subsection{Domestic Research Status of Wetland Models}

Our database is mainly used in the fields of engineering, machinery, road and railway building, petroleum and the flood control. For instance, Wang and his colleagues developed Windows-based platform on the basis of objectoriented systems Die CAD engineering database systems to meet the requirements of mold design, assembly management, device management and mold design results management. Engineering CAD systems solve the problem of low efficiency and facilitate the development and maintenance of data processing engineering CAD software. Jing Hongrong used SQL Server2000 as the backend database, employed Java as Web Service development tool to support the development of subway and car body engineering database system. Jing completed the functional design of reception function queries, visual queries and parameterized queries, effectively managed product development data to support the development of a parallel body engineering project, and shortened the development cycle of vehicle body. Using engineering methods, $\mathrm{Hu}$ Xiaguang established Qinghai-Tibet highway project database, linked the road project to the level of the Qinghai-Tibet Highway to improve the design, in order to ensure smooth traffic and reduce road maintenance costs. Nanjing Railway Survey and Design engineering database system involves all areas and phases of the Railway Survey and Design. The system research and development is conducted to achieve a package of survey data from the storage of archive query of the whole process, to better meet the requirements of the station before and after the integration of professional CAD design. [3] Chen Shutang and other scholars established logging class exploration database, which provides a feasible solution for the field of engineering geology department from the perspectives of database structure design, logging data processing and format conversion. It sets the sum of starting entry techniques through building a database logging class exploration database, and provides a better channel to make full use of data entry logging information resources.

\section{THE ESTABLISHMENT OF WETLAND GIS MOD- EL}

\subsection{The Definition of GIS}

GIS means Geographic Information System. Its development and application in environmental projects provide important advanced means for wetlands protection. It has merits of rapid, real-time spatial information acquisition, analysis capabilities in global resources change and environment dynamic monitoring, as well as disaster monitoring and prevention. GIS has become one of the hot issues which draws more and more attention. GPS precise positioning function, combines with the application of three powerful GIS data and spatial analysis collection, to provide important, advanced technique for the protection of wetlands. [4]

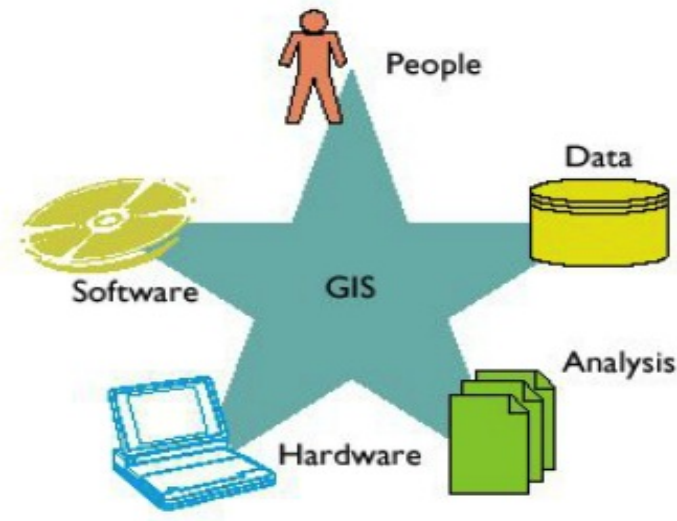

Fig. (1). GIS system.

Geographic information system is composed of computer hardware, software, and different methods of system. The system is designed to support spatial data collection, management, processing, analysis, modeling and display, in order to solve the problem of complex planning and management. (Fig. 1) It is the collection of computer science, geography, geometry, surveying and mapping of remote sensing, wetland science, space science and many other fields. [4] Wetland science involves complex and open system of the earth's surface and the geographic information system. For the study of the movement and transformation pattern of matter and energy in real geographical space, it provides a convenient and accurate space management and spatial analysis method. It can also be applied to other fields such as wetland management, wetland monitoring, wetlands engineering and geochemistry, wetland planning and the evaluation of wetland effects. [5] Through analyzing the spatial distribution of information, monitoring information changes at different times, comparing different spatial data set or other kinds of information, and implementing spatial information and other types of information management, geographic information system makes the abstract and boring data become vivid, intuitive and easy to understand information.

\subsection{Wetland GIS Model}

GIS model mainly includes three steps: wetland data acquisition, data processing and storage, data output. Data acquisition means the collection of wetland information, such as remote sensing data, GIS geographic information, parameter form, parameter information and so on. Data processing and storage is the core of the model. Data analysis predicts the change trend. Concrete model is shown in Fig. (1).

\subsection{Data Acquisition and Input}

Data acquisition system in the data processing system means to take down original data outside the system to the system, and convert the data format outside the system to data format inside the system in order to facilitate processing process. It can realize a variety of ways of data input from data comes with a variety of forms and sources of information. Data acquisition mainly deals with four kinds of data 
input, including graphic data input, such as network graph input; raster data input, such as remote sensing image input; measurement data input, such as Global Positioning System (GPS) data input; and attribute data input, such as the input of numbers and words. [6]

\subsection{Data Editing and Updating}

Data editing and attribute editing mainly include data and graphics. The graphics editing includes topology, graphics, editorial, graphics, grooming, map matching, graphics transformation, projection transformation such as error correction and function attributes editing to complete [7] the database management. Attribute editing mainly deals with database management; it has functions of modifying, adding, deleting and others [7].

Data updating uses new data items or records to replace corresponding data items or records in the data file or database. It inserts data through deleting, modifying, and a series of operations. Because of the time series in the development of space entities, the access to data only reflects the characteristics of data in a certain moment or under a certain time frame. Data changes over time. Data updating can satisfy the needs of dynamic analysis of the occurrence and development of natural phenomena and make regular forecasts. [8]

\subsection{Data Storage and Management}

Data storage is to put data in some formats within or outside the record into the computer storage medium. Its storage is associated with the structure of data file. Storage is the key to establish logical sequences of records, which determine the storage address and can improve the speed of data access. [6] Attribute data is generally managed through relational database. Related commercial database software, such as SQL Server, Fox Base and FoxPro, can be used directly. Spatial data management is the core of GIS data management. It has a variety of graphics and image information with strict logical structure in spatial database.

\subsection{Spatial Query and Analysis}

Spatial query and analysis function include data manipulation operations, data query retrieval and comprehensive analysis. Data query and retrieval find or select the required data from data files, database or storage device. GIS can analyze various phenomena and the process area.

For database output, the basic statistics include:

(1) The overall classification accuracy. [8] Divided by the total number of correct classification reference, the number is calculated as follows:

$$
P_{n}=\sum_{k=1}^{n} p_{k k} / p
$$

(2) The accuracy of the producer. The percentage of the reference data is correctly classified; it is calculated as:

$p_{i j}=p_{i i} / p_{i}+\mathrm{i}$
(3) User accuracy. In same classes, the number is correctly classified as a percentage of the total, representing that the element can be classified as true representatives of probability. The formula is:

$$
p_{j i}=p_{i i} / p_{i}+j
$$

(4) Kappa coefficient is a classification accuracy of statistical values with range between 0 and 1 . This classification indicates that compared with random use, each pixel assigned to any one class will be much better. The formula is:

$$
K=\frac{P \cdot \sum_{k=1}^{n} P_{K K}-\sum_{i=1}^{n}\left(p+i \cdot p_{i}+\right)}{p^{2}-\sum_{i=1}^{n}\left(p+i \cdot p_{i}+\right)}
$$

This function is an independent research issue of GIS; its main characteristic is to help researches to determine the new space relationship between geographical elements. It has become an important symbol which not only differs from other types of system, but also provides users with the flexibility to solve problems of all kinds of special tools.

\subsection{Data Update}

Data updating uses new data items or records to replace corresponding data items or records in data file or database through a series of operations like deleting, modifying and inserting. [9]

\subsection{According to the Output}

According to data, output is the intermediate process and the final result of the process. It includes the editing of digital and graphic data, and the analysis of the operation process of the display. It usually selects the object and the form of display in human-computer interaction. For graphic data elements based on the amount of information and intensity, zoom in and out can be chosen. [8]

Data output usually has the following forms: to visualization through computer screen, plotter or printer output; realize remote transmission through the Internet. [6] In addition, GIS can not only output the total factor maps, but also can output various thematic map with all kinds of statistical figure, charts and data according to user needs.

\section{WETLAND GIS MODEL ANALYSIS}

\subsection{Wetland Monitoring}

Wetland monitoring means to determine the quality of wetland (or pollution) and its change trend on the basis of determining the representative factors that influence the quality of the wetland. Wetland monitoring results data should be accurate and complete response to the time and spatial distribution of pollutant (including pollution emissions) within the scope of an area. From this perspective, monitoring requires data with representative, completeness, accuracy, precision and comparability. [10] 
Wetlands monitoring and the characteristics of wetland pollution lead to following characteristics of data acquisition and processing:

Space: Monitoring spatial entities at a certain point; wetlands have more than $85 \%$ related information to the spatial location.

Timeliness: Data about wetland factors and pollution emissions of pollutants change over time. Wetland monitoring should take time changes into consideration, so the wetland monitoring data needs a fast, accurate and real-time processing process.

Diversity: On one hand, there are many kinds of wetland information sources, including monitoring data, statistical data, graphic data, remote sensing data from different source datas, and compound data; on the other hand, indicators reflect the quality of wetland pollution also vary. For instance, main pollutants reflect atmospheric air quality including sulfur dioxide, total suspended particulate matter, inhalable particles, nitrogen oxides, nitrogen dioxide and so on. Thus, the monitoring data should comprehensively consider the correct connotation of indicators from different categories.

\subsection{The Characteristics of Wetland GIS Model Is Ap- plied to Wetland Monitoring}

GIS can be used in the field of wetland monitoring. The characteristics of GIS itself and demands of wetland monitoring make them inseparable.

According to the functions of GIS, its wide application in wetland monitoring can be analyzed and summarized. Its applications have the following features [8]:

1) Wetland GIS model can realize effective management of wetland monitoring data

The main results of wetland monitoring is the data. The continuity of monitoring makes huge amount of data. Every year, tens of thousands of wetland monitoring data is recorded. It is difficult to ensure the consistency and accuracy of the vast amounts of data if we rely on manual management. It is necessary to establish a wetland monitoring database. The traditional database structure cannot express and store information very well, and it cannot achieve the spatial data management and analysis of spatial information. To solve this problem, geographic space information must be combined with the reality of wetlands.

The GIS model of certain geographical elements in the region can organize and store a collection of related data together in certain organizational way. GIS database contains a large amount of data; among these data, spatial data and attribute data are connected, while spatial data possesses the characteristics of topology. Therefore, GIS database management function can be used to monitor wetlands. Besides, DBMS functions related to the attribute data monitoring also includes the monitoring of spatial data management technology (Fig. 3). For example, information of wetland monitoring and its factors retrieve from the space position; data retrieval can also be achieved from aspects of wetland quality control, conditions monitoring, locations and so on.

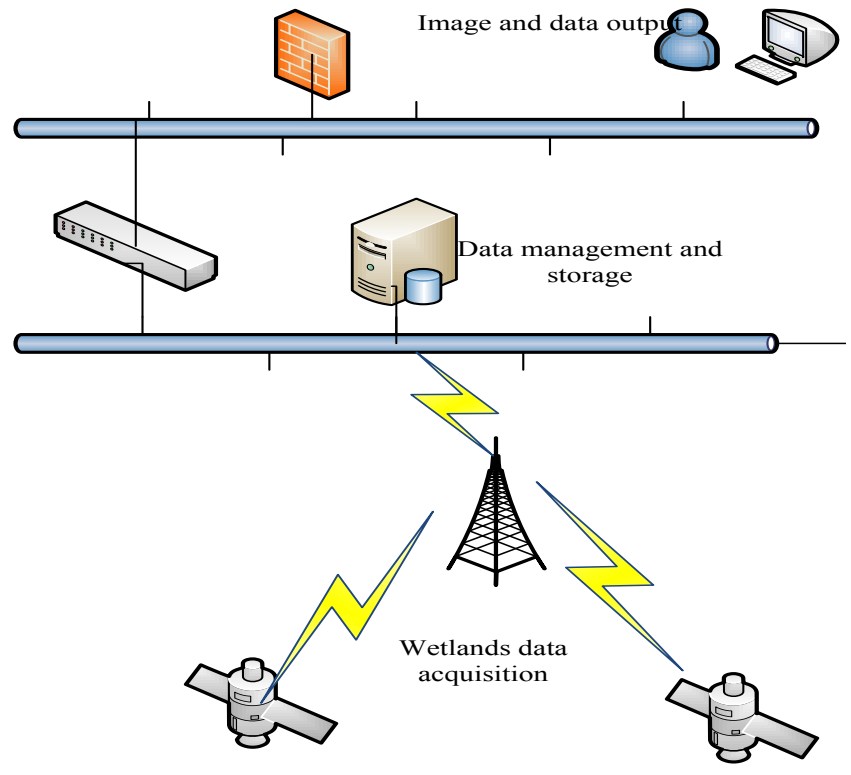

Fig. (2). Wetland GIS model implementation of wetlands monitoring.

\section{2) GIS vivid graphics expression}

GIS has very powerful graphics display and mapping functions. But it is not only a map tool; it is also a combined geographic information analysis system which has functions of wetland pollution monitoring, wetland management, as well as geographical characteristics and wetland factors analysis. Maps, tables and graphs make data expression visualized; dynamic coupling, management and query make a lot of abstract, boring data become vivid, intuitive and easy to understand. Using electronic map, images can intuitively interact with users, while users can process data relying on their inherent humanly understanding of nature and logic. Especially in recent years, with the GIS application and development of virtual reality technology, spatial distribution provides accurate and intuitive visual display on wetland pollution diffusion patterns; real-time, dynamic displays on wetland status changes are also accessible. [11]

\section{3) Composite analysis function of GIS}

Computer technology combined with spatial data of GIS includes the processing of spatial information with all kinds of advanced features. But its basic functions are data acquisition, management, processing, analysis and output. Based on these basic functions of GIS, the technology of spatial analysis can realize model analysis and data integration through network technology and database technology, which deduces rich and colorful applications, such as secondary development function.

GIS has such powerful functions on spatial data management and comprehensive analysis of spatial data. Meanwhile, above $85 \%$ of wetlands information is related to space. Thus, the management of wetland monitoring data has more advantages. The combination of GIS and wetland model provides a more efficient and professional processing method. Therefore, using the powerful spatial analysis ability of 
GIS technology to realize the wetland information management is reasonable and necessary.

\subsection{Progress in the Application of GIS in Wetland Moni- toring}

Wetlands monitoring depends on the acquisition and processing of information, and more than $85 \%$ of the wetlands information is related to the spatial location. Thus, the geographic information system is an effective wetland monitoring tool. GIS has powerful spatial analysis and data processing functions, which help GIS to make full use of the function modules of selected wetlands monitoring model. Through multi-source information processing, the dynamic changing patterns of wetland can be found, while the dynamic monitoring of wetland can be realized and represented by vivid graphs and pictures.

1) The GIS application in wetland monitoring data management analysis

The greatest characteristic of GIS is that, it investigates and analyzes the geographic distribution of the whole or part of the earth surface (including the atmosphere) through data collection, storage, management, operation, analysis and visualization expression. It can draw scientific conclusions on the basis of existing spatial and attribute information through data processing and management. These characteristics help it to combine with wetland monitoring. In various wetlands, GIS make wetland management process and spatial analysis operation more convenient, make interactive interfaces more friendly, intuitive and vivid shows. It undoubtedly promotes the development of wetland monitoring technology. [7]

GIS application in wetland monitoring data management should be analyzed from the aspects of wetland information storage, wetlands mapping, map display, and the simulation and analysis of complex conditions.

According to different aims, wetland monitoring can be divided into wetland quality monitoring, pollution source supervision and monitoring and emergency monitoring. GIS application can be used in wetland monitoring data management for further instructions. From the angle of GIS spatial analysis and comprehensive analysis, the application of GIS can be illustrated as: [9]

Wetland quality monitoring is the main part of the monitoring work. Each element which is related to wetland pollution and the tendency of pollutants should be monitored; and evaluation effects of control measures and judging standards should also be used to accelerate the improvement of wetlands. The accumulation of quality monitoring data determines the status and development trend of wetland pollution in this region. Using GIS trend prediction model, scholars can make accurate forecast on the changing trend of wetlands. For example, information on an area of wetland in 2000 is shown in Fig. (3). Through analysis of information, GIS wetland model can clearly represent the information of wetland in 2010, as shown in Fig. (4).

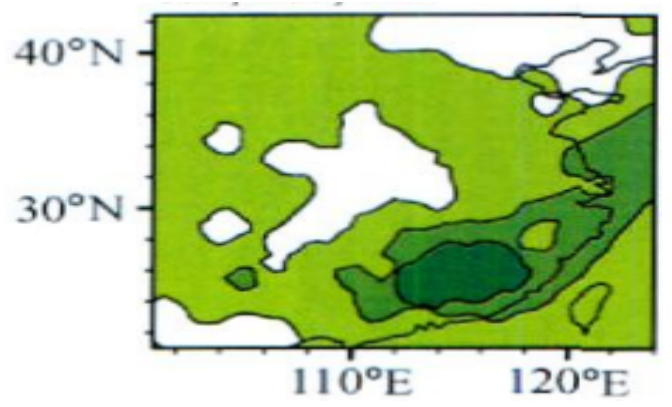

Fig. (3). Wetland information of 2000.

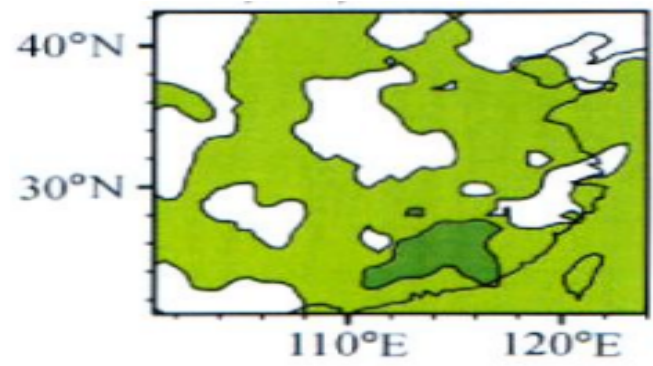

Fig. (4). Wetland information of 2010.

\section{CONCLUSION}

Geographic information system has the property of spatial and non-spatial data management and the ability of spatial analysis. It cannot be replaced by any other tools. Through editing and transforming a variety of spatial and statistical data, GIS can establish the topological relation of different spatial objects in points, lines and faces. The four different kinds of spatial data are expressed as various thematic maps and charts with layered output. It saves a lot of manpower, material and financial resources. What's more, it can obtain results with high speed and high accuracy, and realize the wide range of application in wetland monitoring. With the continuous development of GIS technology and its application of wetland monitoring, it can be predicted that GIS application in wetland science will be further exploited and improved. [11] In the future, the application of GIS in wetland monitoring model should be developed in combination with national information promotion. Based on related electronic projects, GIS in wetland monitoring models should be specialized and used in data management process, in order to promote the development of wetland assessment, supervision and tracking analysis.

In this paper, GIS technology is used to build the analysis model for wetland monitoring, and stress the importance of information technology. But only with the combination of information technology and the actual research, and under the premise of pursuing accuracy, can we actually use the computer model effectively.

\section{CONFLICT OF INTEREST}

The authors confirm that this article content has no conflict of interest. 


\section{REFERENCES}

[1] I. Vander, and C. J.Sande, "A segmentation and classification Approach of IKONOS-Imagery for land cover mapping to assist flood risk and flood damage assessment," International Journal of Applied Earth Observation and Geoinformation, vol.4, no. 3, pp.217-29, 2003.

[2] E.M. Patrick, "Theory and methods for modeling and evaluating multiscale landscape structures," International Archives of Photogrammetry and Remote Sensing, vol. 78, pp. 120-123, 2001.

[3] L. B. Bronge, and B. Naslund-Landenmark, "Wetland classification for Swedish CORINE Land Cover adopting a semi-automatic interactive approach", Canadian Journal of Remote Sensing, vol.28, no. 2, pp. 139-155, 2002.

[4] X.Y. Zhou, and W. Xu,. "Phytoremediation in water environment ecological restoration", Environmental Bulletin, vol.35, pp. 24-28, 2011.
[5] D.S. Wang and H.W.Tan, "The impact of human activities on the river ecosystem", Science Technology and Engineering, vol. 4, pp. 298-310, 2010

[6] M.G.H. Omran, and M. Mahdavi, "Objectives and Principles of river ecosystem restoration", Applied Mathematics and Computation, vol. 198, pp. 19-31, 2012. (In Chinese)

[7] Zhu Sh., "Technical Overview of river restoration", Guangdong chemical,vol.40,pp135-136, 2013.

[8] Wen Y.W, Lin H.X, Lan X.Zh, "Usage of biological floating bed technology for water restoration research and demonstration", Beijing Water Conservancy, vol.6, pp20-22, 2003.

[9] Y.H. Wang, and W.P, Cao, "Ceramic biofilm process for municipal wastewater COD and ammonia", Water Treatment Technology, vol. 35, pp67- 69,2009.

[10] J.C. Zhong, F.S.Liu, "Environmental effects of lake sediment dredging", Lake Science, vol.21, pp.335-344,2009.

[11] J. Wang, "Plants gravel bed constructed wetlands wastewater treatment technology and my Project", Beijing: China Environmental Science Press, pp.212-220, 2009.

Received: May 26, 2015

Revised: July 14, 2015

Accepted: August 10, 2015

(C) Liwei Liu; Licensee Bentham Open.

This is an open access article licensed under the terms of the (https://creativecommons.org/licenses/by/4.0/legalcode), which permits unrestricted, noncommercial use, distribution and reproduction in any medium, provided the work is properly cited. 